\section{Remarks on Acoustic Oscillations in a Solid Propellant Rocket}

\author{
F. E. C. CULICK* \\ California Institute of Technology, Pasadena, Calif.
}

$\mathbf{T}$ HE problem of unstable pressure oscillations in solid propellant rocket chambers has been a subject of much work in recent years. Of the various kinds observed, perhaps the most common and simplest problems to describe are those related to classical acoustic modes for a closed chamber. It is somewhat surprising that the best, and indeed quite complete, data reported by Brownlee and Marble, ${ }^{1}$ for small cylindrical rockets, have received relatively little attention. The only published quantitative interpretation seems to be that of Bird, et al. ${ }^{2}$, who speculated that the main source of damping was the action of particles suspended in the combustion products. It then is possible to offer an explanation for the observed linear stability boundary, reproduced here in Fig. 1. Namely, the locus of neutral stability for waves in the chamber is defined by the balance of energy lost because of particle damping and work done on the waves by the gases in the combustion zone.

Such a description, although possible and perhaps adequate in some cases, fails to explain the obvious shift of the stability boundary with length. Moreover, one ought to explain why the frequency of the observed standing wave mode is not very different from that calculated according to the classical formula, ${ }^{1}$ and why, in fact, the same mode was found in all (400) rockets fired. This information may be obtained by examination of the problem formulated as one of acoustics, but with perturbations proportional to the mean flow Mach number. One then finds formulas for the real and imaginary parts of the frequency. The first shows that the frequency is altered by a negligible amount, from its classical value, by the action of the burning surface. On the stability boundary, the formula for the imaginary part [the attenuation constant $\lambda$ in $\exp (-\lambda t)]$ vanishes, giving the formula for $K_{n}$, the ratio of burning surface area to nozzle throat area,

$$
K_{n}=\left(2 \Gamma \bar{a} / \gamma^{1 / 2} D_{p}\right)\left[A_{b}{ }^{(r)}+m_{1}^{2} /\left(1-m_{1}^{2}\right)(\beta+\sigma)\right]
$$

where $\bar{a}$ is the speed of sound and $\Gamma$ is the function of $\gamma$ appearing in the characteristic speed $c^{*}$. The real part of the admittance function of the burning surface is $A_{b}(r), \beta$ is the damping constant associated with particles, and $\sigma$ represents damping at the head end of the rocket. The term containing $m_{1}=m / \boldsymbol{\varkappa}_{m n}$ contains the direct effects of mean flow (convection of energy and work); $m$ and $\varkappa_{m n}$ are separation constants in the eigenfunction $\cos (m \phi) J_{m}\left(\boldsymbol{\kappa}_{m n^{r}}\right) \cos \left(k_{l} z\right)$. For the oscillations observed by Brownlee and Marble, $k_{l}=0$, $m=1$, and $\varkappa_{01}=1.84$.

Now with the size range of particles assumed in Ref. 1, $\beta \propto D_{p}{ }^{-2}$ so that if $\sigma=m_{1}=0$ and $A_{b}(r)$ is taken to be constant, $K_{n} \propto D_{p}$, in accord with Fig. 1 ; reasonable numerical values can be chosen so as to fit the boundary exactly. ${ }^{2}$ When axial motions are absent $\left(k_{l}=0\right)$, the exhaust nozzle has no influence on the waves in the chamber and this interpretation fails as remarked before.

An alternative is to set $\beta=0$, and suppose, as an estimate,

\footnotetext{
Received November 5, 1965.

* Assistant Professor of Jet Propulsion, Guggenheim Jet Propulsion Center. Member AIAA.
}

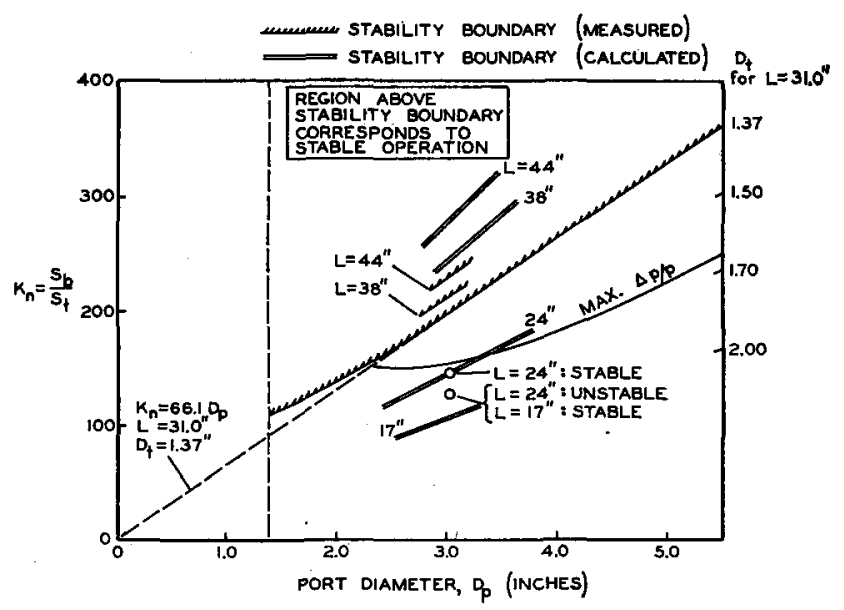

Fig. 1 Stability boundary, 101 mode (Ref. 1).

that $\sigma$ is nonzero because of simple viscous shear acting between the oscillations and the head end of the rocket; then $\sigma=21.4 / L D_{p}^{1 / 2}$. If the mean flow is taken into account, then set $m_{1}=0.543$ in the numerator of $K_{n}$. The stability boundary for $L=31.0$ in. may be matched by choosing values of $A_{b}{ }^{(r)}$ variable with $D_{p}$ but consistent with measured values (e.g., Ref. 3); they are slightly low because $\sigma$ undoubtedly is underestimated. More importantly, the predicted translation of the boundary agrees quite well with the measurements.

It happens that the mean flow acts to drive this mode ${ }^{4}$ and this action is stronger for higher modes. However, as the frequency increases, $A_{b}(r)$ rapidly becomes negative; the burning process then absorbs energy from the waves. The balance between the burning and the effects of the mean flow is such that the only mode sustained is that observed.

Similar conclusions may follow for other instances of small amplitude oscillations in solid rockets. Even though the admittance function $A_{b}(r)$ (which represents essentially $p-v$ work done by the combustion region on the waves) is unknown, it might be estimated fairly well from data for known propellants. However, it must be emphasized that the propellant used by Brownlee and Marble contained no aluminum and hence, there probably were no particles of significant size or number in the gas phase. If there is aluminum in the propellant, the formation of particles easily can lead to values of $\beta$ much larger than $\sigma$. The appropriate energy balance then includes, at least, the effects of burning, the mean flow, and the particles.

\section{References}

1 Brownlee, W. G. and Marble, F. E., "An experimental investigation of unstable combustion in solid propellant rocket motors," AIAA Progress in Astronautics and Rocketry: Solid Propellant Rocket Research, edited by Martin Summerfield (Academic Press Inc., New York, 1960), Vol. 1, pp. 455-494.

${ }^{2}$ Bird, J. F., McClure, F. T., and Hart, R. W., "Acoustical instability in the transverse modes of solid propellant rockets," 12th International Astronautical Congress (Academic Press Inc., New York, 1963), pp. 459-473.

${ }^{3}$ Horton, M. D. and Price, E. W., "Dynamic characteristics of solid propellant combustion," Ninth Symposium (International) on Combustion (Academic Press Inc., New York, 1963), pp. 303-310.

4 Cantrell, R. H. and Hart, R. W., "Interaction between sound and flow in acoustic cavities: Mass, momentum, and energy considerations," J. Acoustic. Soc. Am. 36, 697-706 (April 1964). 